\title{
Cross-modality matches of finger span and line length
}

\author{
CLAYTON L. VAN DOREN \\ Case Western Reserve University, Cleveland, Ohio
}

\begin{abstract}
Perceived finger span-the perceived spatial separation between the tip of the thumb and the tip of the index finger-was measured by using cross-modal matching to line length. In the first experiment, subjects adjusted finger span to match the length of line segments presented on a video monitor, and conversely, with both hands. Subjects also made estimates of finger span in physical units ("dead reckoning"). Finger spans were measured by using infrared LEDs mounted on the tip of the thumb and the finger tip, so the hand made no contact with any object during the experiment. Unlike in previous studies, the results suggest that perceived finger span is proportional to line length and slightly shorter than the actual span, provided that corrections are made for regression bias. The effect of finger contact was assessed in a second experiment by matching line length both to free span and to spans constrained by the pinching of blocks in the same session. The matching function when subjects were pinching blocks was accelerating, consistent with previous reports. In contrast, matched line length was a decelerating function of free span. The exponent of the free span matching function in the second experiment was slightly smaller than in the first experiment, probably due to uncorrected matching biases in the second experiment.
\end{abstract}

A person usually has no difficulty reaching for a glass of water and picking it up. It can be done even with the eyes closed after a couple of tries. There is, obviously, exquisite motor control based on visual and somatosensory information. But do the different sensory modalities tell us the same thing? For example, does the glass look as big as it feels? The question does not seem that interesting at first - surely the perceived size of the glass does not depend on whether it is looked at or is held in the hand. Experimentally, though, the answer depends on how the equivalence of size is measured. Magnitude estimates of span are nearly linear with respect to the actual distance between the tips of the index fingers of two hands held apart (Gogel, Wist, \& Harker, 1963; Stanley, 1966; M. Teghtsoonian \& R. Teghtsoonian, 1965). But the bulk of the experimental evidence suggests in contrast that the span perceived between the tips of the thumb and index finger of the same hand is a nonlinear function of the actual distance, is not veridical, and is not commensurate with perceived visual size.

Jastrow (1886) provided the first such evidence by having subjects adjust finger span by sliding a movable carriage held between the thumb and index finger to match the length of a viewed line $(0.5-12 \mathrm{~cm})$; or, to se-

The author would like to thank Robert Teghtsoonian and Calvin Garbin for reviewing the manuscript and making many helpful comments and suggestions; Purwanto Suwondo, Lisa Menia, Jennifer Evans, and Michael Haines for technical assistance and data collection; and Robert Montz for making the blocks used in the second experiment. This work was funded by Grant NS27958 from the National Institutes of Health. Correspondence should be addressed to C. L. Van Doren, MetroHealth Medical Center, H601, 2500 MetroHealth Drive, Cleveland, OH 44109-1998 (e-mail: clv2@po.cwru.edu). lect a predrawn line from a set or mark a length on ruled paper to match the width of blocks held between the thumb and the index finger. The results are plotted in the upper panel of Figure 1, which shows the value of the match as a percentage of the actual size of the reference. As in the original graph (Jastrow, 1886, Figure 5), the ordinate for percentages greater than $100 \%$ is linear, but the ordinate for ratios below $100 \%$ is logarithmic. Subjects overestimate line length by adjusting finger span (closed points) and underestimate finger span by adjusting line length (open points). In other words, spans are perceived as being shorter than lines of equal physical length.

Jastrow's data (1886) are replotted in the lower panel of Figure 1 after converting the relative measures into absolute distances to produce conventional cross-modal matching (CMM) functions. The data are fit reasonably well by power functions of the following form:

$$
y=k x^{\varepsilon} \text {. }
$$

The function for line length matched to finger span ( $L \rightarrow S$, open points) has a scale $k$ of 0.45 (for distances in centimeters) and an exponent $\varepsilon$ of 1.3. R. Teghtsoonian and $M$. Teghtsoonian (1970) performed the same analysis of Jastrow's data with nearly identical results: $k=0.43$, $\varepsilon=1.3$. The complementary $S \rightarrow L$ function has a scale of $k=2.3$ and an exponent of $\varepsilon=0.69$. The algebraic inverse of the $S \rightarrow L$ function, as plotted in the lower panel of Figure 1 (closed points), has a scale of 0.29 and an exponent of 1.5 , in good agreement with the direct $L \rightarrow S$ results (see the Appendix).

Mashhour and Hosman (1968) similarly did direct crossmodal matches of finger span (measured with the thumb fixed and the index finger attached to a sliding indicator) 
to the length of line segments $(1.5-15 \mathrm{~cm})$, producing a function that was slightly curved in $\log$-log coordinates. The authors fit the data using a power function with a constant:

$$
y=k(x-c)^{\varepsilon},
$$

with values of $c=-1.5 \mathrm{~cm}$ and $\varepsilon=0.73$ (no value of $k$ was given). Stevens (1969) objected to the use of the constant solely to improve the fit (i.e., without a sound rationale for the existence of a threshold) and fit the same data (though not as well) with a conventional power function with an exponent of 0.52 . In either case, the $S \rightarrow L$ function is decelerating, consistent with the results from Jastrow (1886).

Stevens and Stone (1959), rather than using cross-modal matching, measured psychophysical functions by using magnitude estimation (ME) of finger span produced by the holding of blocks between the thumb and the middle finger $(0.23$ to $6.37 \mathrm{~cm})$. Results with two different moduli yielded exponents of 1.33 . Using similar procedures (the holding of blocks between the thumb and the index or middle fingers), Mashhour and Hosman (1968), R. Teghtsoonian and M. Teghtsoonian (1970), and Jones (1983) all measured accelerating ME functions with exponents in the range from 1.1 to 1.3 , in agreement with cross-modal matching results (see the Appendix, Equation A3).

Although not a study of finger span per se, subjects in the experiments of Ekman, Berglund, Berglund, and Lindvall (1967) and Berglund and Olsson (1993) used finger span to indicate perceived odor strength. Finger span was measured with the thumb fixed and the index finger in a sliding carriage attached to a linear potentiometer. As part of a "calibration" protocol, subjects were asked to generate finger spans at fixed percentages of their maximum span-a variant of more traditional magnitude production procedures. These functions were then inverted and used to convert the finger spans generated in response to olfactory stimuli into normalized numerical values. The results from Ekman et al. (1967, Figure 2), as well as the unpublished data from Berglund and Olsson (1993, personal communication), are plotted in Figure 2, with the target percentage on the ordinate and the resulting span on the abscissa. Each thin line is the average of 10 trials from 1 of 16 subjects ( 8 from each study). ${ }^{1}$ The points are the averages across all subjects, and the thick line is the best-fitting power function, which has an exponent of 0.83 . The power function is constrained to go through the point $(100 \%, 100 \%)$, since there was a rigid stop placed at each subject's maximum span. These data suggest that perceived finger span (in terms of the percentage of the maximum span) is a decelerating function of the actual finger span.

All the studies described above required that the finger and thumb make contact with an object either to set the fingers to a known span or to measure a span as a matching variable. In contrast, Chan, Carello, and Turvey (1990) studied the differences between perceived span when the fingers contacted an object and when they did not. I will refer to the latter specifically as free span, or $S_{\text {free }}$. Sub-
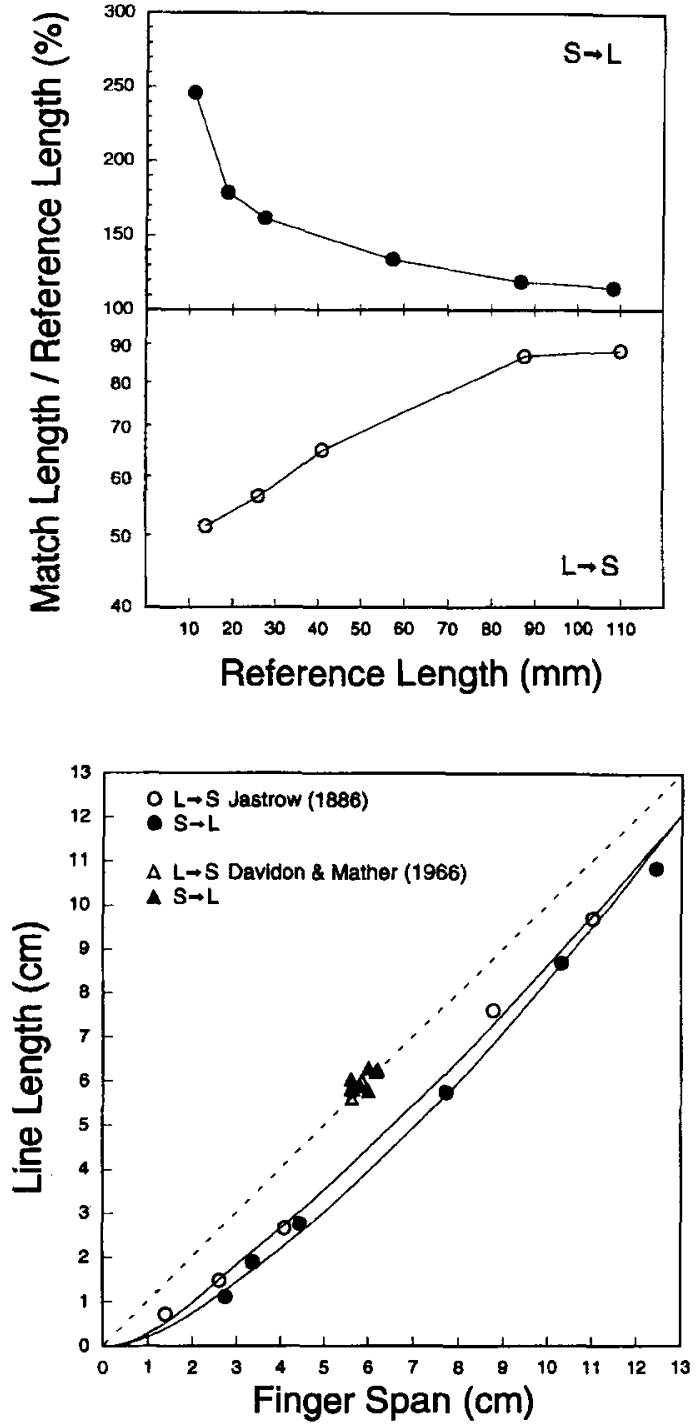

Figure 1. (Top) Cross-modal matching data replotted from Jastrow (1886, Figure 5). Each point represents the relative error made when one is matching finger span to the length of a viewed line segment (filled points) or the converse (open points). Each point is the average from about 30 subjects. The matches are expressed as the ratio of the matched length to the reference length in percent. Note that the ordinate for matches less than $100 \%$ is logarithmic, whereas ratios greater than $100 \%$ are plotted linearty, as in the original figure. (Bottom) Cross-modal matches with finger span on the abscissa and line length on the ordinate. The circles are the same data from Jastrow (1886) plotted in the top panel, and the triangles are matches derived from simultaneous category ratings (from "Cross-Modal Judgments of Length," by R. S. Davidon and J. H. Mather, 1966; American Journal of Psychology, 79, pp. 409-418, Tables 1 and 3. Copyright 1966 by the Board of Trustees of the University of Illinois. Used with permission of the University of Illinois Press). Open points are from $L \rightarrow S$ matching, and closed points are from $S \rightarrow L$ matching. The latter functions have been irverted.

jects used free span, measured with a ruler, to match the width of blocks perceived visually or held between the index finger and the thumb of the opposite hand. In both cases, the free span was a decelerating function of block 


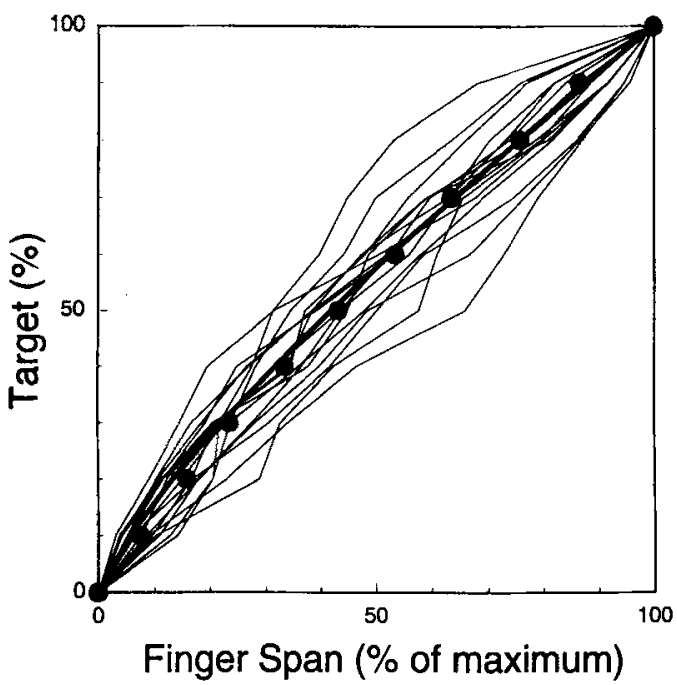

Figure 2. Finger span magnitude production data from Ekman et al. (1967) and Berglund and Olsson (1993, and personal communication). Subjects were asked to generate finger spans $(S$, plotted on the abscissa) that were fixed target percentages of their maximum $(N$, plotted on the ordinate). Each thin line is the average of 10 repetitions from 1 subject, and the points are the average responses across 16 subjects. The thick line is the best-fitting power function to the data: $S=k N^{12}$. (Data are in part replotted from "Perceived Intensity of Odor as a Function of Time of Adaptation," by G. Ekman, B. Berglund, U. Berglund, and T. Lindvall, 1967, Scandinavian Journal of Psychology, 8, pp. 177-186, Figure 2. Copyright 1967 by Scandinavian University Press. Adapted by permission. Data from Berglund and Olsson, 1993, are copyright 1993 by the Psychonomic Society, Inc.)

size (for held blocks, $k=1.5, \varepsilon=0.82$; for seen blocks, $k=1,8 ; \varepsilon=0.75)$. Inverting the function relating free span to visual block width yields, in effect, a "line length" to free span function with an exponent of 1.3 in agreement with the results described above. It is also interest- ing (and will be of particular importance later) that free span and span when subjects were touching blocks were not perceived equally. Rather, free span was an accelerating function of span perceived when subjects were touching blocks.

The matching and estimation exponents from these previous studies are summarized in Table 1. All the functions are nonlinear, and all but the results from Ekman et al. (1967) and Berglund and Olsson (1993) indicate that perceived finger span is an accelerating power function of the actual span, with an exponent in the range of 1.1-1.5. (Note that the reciprocal of the exponents for functions in which finger span was matched to line length or number have been reported in Table 1, consistent with reporting all functions as if matches were made to finger span.)

There is some evidence from two other experiments, however, which suggests that perceived finger span is at least linear with, and perhaps equal to, perceived line length. First, Jones (1983) measured perceived finger span by using magnitude estimation (as cited above), but also used a 20-point category scale to rate the combined thickness of two blocks pinched between the thumb and the index finger of both hands.

Estimation yielded accelerating functions (Table 1), but the category judgments of combined thickness were fit best by a linear additive model (Anderson, 1974). Visual estimates were also linear and additive. Jones (1983) argued that the linear psychophysical functions obtained with functional measurement are free from the biases that have been attributed to magnitude estimates (see, e.g., Poulton, 1979) and that may have produced the nonlinearities observed in other studies.

Second, Davidon and Mather (1966) asked subjects to make simultaneous category judgments of the length of bars either viewed through a frame or held between the thumb and the index finger. The judgments were made for one of four standard bars $(5.6,5.8,6.0$, and $6.2 \mathrm{~cm})$

Table 1

Exponents and Seales for Finger Span Psychophysical Functions

\begin{tabular}{|c|c|c|c|c|}
\hline Study & Task* & Exponent $\dagger$ & Span Method & Finger \\
\hline \multirow[t]{2}{*}{ Jastrow (1886) } & $S \rightarrow L$ & $(1.5)$ & carriage & index \\
\hline & $L \rightarrow S$ & 1.3 & blocks & index \\
\hline Stevens \& Stone (1959) & $N \rightarrow S$ & 1.3 & blocks & middle \\
\hline \multirow{4}{*}{ Mashhour \& Hosman (1968) } & $N \rightarrow S$ & 1.2 & blocks & index \\
\hline & $N \rightarrow S$ & 1.2 & blocks & index \\
\hline & $N \rightarrow S$ & 1.3 & blocks & index \\
\hline & $S \rightarrow L$ & $(1.4)$ & carriage & index \\
\hline R. Teghtsoonian \& M. Teghtsoonian (1970) & $N \rightarrow S$ & 1.2 & blocks & middle \\
\hline \multirow[t]{2}{*}{ Jones (1983) } & $N \rightarrow S$ & 1.1 & blocks & index \\
\hline & $N \rightarrow S$ & 1.2 & blocks & index \\
\hline \multirow[t]{2}{*}{ Chan et al. (1990), Experiment 1} & $S_{\text {free }} \rightarrow L$ & (1.3) & free span & index \\
\hline & $S_{\text {free }} \rightarrow S$ & $(1.2)$ & free span $\rightarrow$ blocks & ind $\rightarrow$ ind \\
\hline \multirow[t]{2}{*}{ Chan et al. (1990), Experiment 2} & $S_{\text {free }} \rightarrow S$ & (1.3) & free span $\rightarrow$ blocks & ind $\rightarrow$ ind \\
\hline & $S_{\text {free }} \rightarrow S$ & $(0.90)$ & free span $\rightarrow$ blocks & mid $\rightarrow$ ind \\
\hline \multicolumn{5}{|l|}{ Ekman et al. (1967), and } \\
\hline Berglund \& Olsson (1993) & $S \rightarrow N$ & $(0.83)$ & carriage & index \\
\hline
\end{tabular}

${ }^{*} X \rightarrow Y$ indicates that modality $X$ was matched or assigned to modality $Y$. $S$, finger span produced by touching an object; $S_{\text {free }}$, finger span without touching; $L$, line length; $N$, numerical estimates or targets. $†$ Values in parentheses are reciprocals of the actual exponents, so that all values can be compared as if $N$ or $L$ were matched $t o$ finger span. 
and eight comparison bars. The points of subjective equality (PSEs) between the standard and comparison stimuli, defined by lengths yielding equal category judgments, were not significantly different for the visual and haptic ratings. The PSEs are plotted in Figure 1 (bottom panel) - the open triangles are from the $L \rightarrow S$ experiment, and the closed triangles represent $S \rightarrow L$ data. The PSEs are closely scattered about the line of equality (dashed line), albeit at a single length, whereas the line lengths chosen by Jastrow's subjects were consistently shorter than the finger span.

In sum, cross-modal matching and magnitude estimation produce nonlinear (and typically accelerating) psychophysical functions for finger span, but not for visual line length. Functional measurement (Jones, 1983), in contrast, yields a linear function, and dual-category rating (Davidon \& Mather, 1966) shows that perceived finger span and visual length are nearly equal (at least near $6 \mathrm{~cm}$ ). The latter does not preclude nonlinearity at other lengths, however. Why are the results different? Are the nonlinearities due to biases introduced by the methodology (see, e.g., Poulton, 1979, 1989), or is perceived finger span really nonlinear? Furthermore, is there a difference between perceived span when the fingers are touching an object or not? To address these questions, I chose to follow the general methods of Jastrow (1886), Mashhour and Hosman (1968), and Chan et al. (1990), and use mainly the simplest and most direct comparison of perceived span and length - cross-modal matchingto avoid variability introduced by individual differences in assigning numbers (see, e.g., Collins \& Gescheider, 1989 ), or the tendency of subjects to use numbers corresponding to physical units of length ( $R$. Teghtsoonian \& M. Teghtsoonian, 1970) rather than sensation magnitude. In the first experiment, I also used a method in which the fingers were not in contact with test objects (bars, rods, or blocks) or a measuring device (sliding carriage), eliminating any information based on tactile cues. Also, both span and length were continuously variable by the experimenter or the subject, permitting CMM in both directions and avoiding categorization strategies that could be introduced by discrete stimuli. The results of the first experiment differed from the earlier results summarized in Table 1, most likely because of the use of free span versus touched blocks. In the second experiment, line length was matched to touched blocks and to free span by the same subjects; identical protocols were used in order to identify potential contributions of tactile information.

\section{METHOD}

\section{Experiment 1}

Twelve subjects in their early and mid twenties, 6 of them male and 6 female, completed six experimental sessions for which they were paid. None of the subjects reported sensorimotor abnormalities of any kind. Eleven subjects reported that they were righthanded, and the 12 th subject was left-handed. In two sessions, the subjects matched their free finger span to the length of line seg- ments displayed on a video monitor, with the left hand in one session and the right hand in another; in two sessions, they adjusted line length to match a preset free span, again with both hands; in the fifth session, the subjects repeated their first session, and in the final session, the subjects made direct estimates ("dead reckoning") of finger span in physical units - either centimeters or inches as they chose. The order of the first four sessions was partially counterbalanced, with half the subjects ( 3 male and 3 female) starting with free span-to-line matching $\left(S_{\text {free }} \rightarrow L\right)$ and half with line-tospan matching $\left(L \rightarrow S_{\text {free }}\right)$. In the second session, the subjects performed matches in the opposite direction, but with the same hand, and then they repeated the sequence with the opposite hand. Hand use was not balanced completely. For each direction, 4 subjects started with their left hand, and 2 with the right. The subjects performed the six sessions with variable schedules, with a median intersession interval of 25 days. The shortest interval was 0 days ( 1 subject performed the fifth and sixth sessions on the same day), and the longest was 183 days ( 1 subject, between Sessions 2 and 3).

The subjects rested their forearms on a table placed at a comfortable height at their side. A screen placed at the wrist prevented the subjects from seeing the tested hand during the experiment. The subjects were encouraged to use a comfortable and relaxed posture for the hand, wrist, and arm; they were allowed to move during the rest periods, but they were asked to assume the same posture (as best they could) during the trials. They were also instructed to generate finger spans by using a " $\mathrm{C}$ " posture of the thumb and index finger (as shown in Figure 3), with the other fingers curled loosely. The " $\mathrm{C}$ " posture was demonstrated to each subject, and was used to avoid hyperextension of the thumb interphalangeal joint or excessive flexion of the interphalangeal joint of the index finger. Undesirable postures are also shown in Figure 3. The plane of movement was roughly horizontal, though for some subjects it was tilted away from the body or toward the midline by $10^{\circ}-20^{\circ}$. The exact orientation of the plane was not measured.

Free finger span was measured with a three-dimensional motion analysis system (Selspot II, Selcom Selective Electronic Co., Partille, Sweden) that used four video cameras to track the threedimensional position of two infrared LEDs, one on the index fingertip, and the other on the thumb. The motion analysis system sampled the locations of the LEDs, calculated their separation in real time, and converted the separation distance to a voltage. The voltage was sampled at $10 \mathrm{~Hz}$ by using an analog $1 / O$ board (NB-DIO$16 \mathrm{H}$, National Instruments Corp., Austin, TX) and a microcomputer (Macintosh IIx, Apple Computer Inc., Cupertino, CA), and was converted back to a distance by using calibration data taken with LEDs mounted on the jaws of vernier calipers at the beginning of each session.

The approximate positions of the LEDs is shown in Figure 3. The positions were chosen so that the LEDs were as close as possible (usually less than a centimeter apart) with the palps of the thumb and index finger just touching (i.e., at zero span) but were still emitting roughly perpendicular to the plane of the thumb and forefinger. The LEDs were held in place by using double-sided tape with glove fingertips cut from sized surgical gloves slipped over the ends of the digits. The glove fingertips were carefully chosen to fit snugly, but not to restrict circulation, as judged from the subjects' reports. The thin latex rubber did not degrade the image of the infrared LEDs.

The span measurements were verified by measuring the span while subjects held 1.95-, 3.93-, 5.90-, 7.90-, and 9.99-cm pieces of plastic drinking straws between dots marked on the thumb and the index finger corresponding to the points of contact at zero span. The straws were stiff, but very light, so that the subjects could hold them securely without visible indentation of the fingertips. Four subjects picked up the five straws one at a time, in ascending order, 30 times each. Errors were taken as the difference between the measured span and actual straw length, minus an offset 


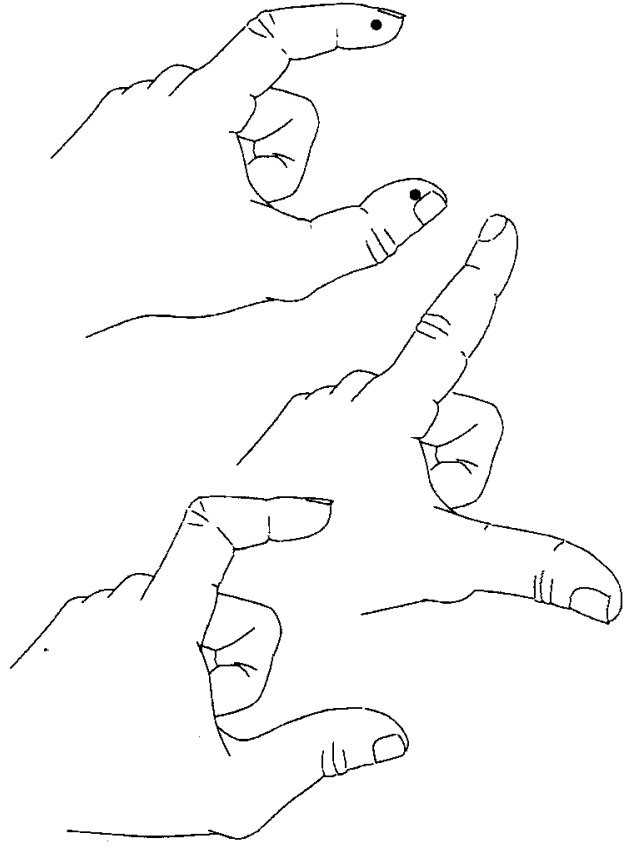

Figure 3. The uppermost drawing shows the proper " $\mathrm{C}$ " posture subjects were instructed to use when producing finger spans. The black dots are approximate locations of the infrared LEDs. The lower drawings show postures that were discouraged.

equal to the average of these differences calculated for each subject. The offset compensated for errors made in the initial measurement of the zero span (see below). The standard deviation of all of the measurement errors across straw lengths and subjects (600 measurements) was $0.21 \mathrm{~cm}$.

The LED separation corresponding to zero span was measured at the beginning of every session by asking subjects to "just touch" the palps of the thumb and the index finger and by recording the distance between the LEDs. The subjects were then asked to open their thumb and finger to a "maximum comfortable span" that maintained the appropriate posture and that they felt they could produce throughout the experiment without getting tired. As expected, the maximum span was correlated to hand size. The latter was gauged roughly by the sum of the distances from the base of the cleft between the thumb and the index finger to the tips of the thumb and the index finger, measured from hand tracings taken from the first session with each subject. The correlation between the median maximum span from the first four sessions and hand size was modest, but significant $\left(r^{2}=.593, p=.041\right)$. Zero spans were measured again at the end of each session, but the recorded span was rarely zero. The distribution of these "re-zero" errors across all subjects and experimental conditions was approximately normal, with a mean of $0.08 \mathrm{~cm}$ and a standard deviation of $0.40 \mathrm{~cm} .^{2}$

The free-span measurement system made it possible to prompt subjects to produce specific spans (as in the $L \rightarrow S_{\text {free }}$ experiment) without using a set of grasped objects. The actual span was measured and compared with a desired target, and one of seven LEDs in a "null meter" was then turned on, depending on the size of the error. The central LED (green) was turned on if the finger span was within $0.12 \mathrm{~cm}$ of the target span. Larger errors turned on successive LEDs - first a yellow LED (error range $\pm 0.12-0.36 \mathrm{~cm}$ ), then one of two red LEDs (error range $\pm 0.36-0.84 \mathrm{~cm}$, and $\pm 0.84-1.80 \mathrm{~cm}$, respectively) to either side of the central LED. Positive errors (spans that were too large) could be displayed by LEDs on either the right or the left side of the meter, depending on the subject's preference.
The subject's task was to achieve and maintain a finger span that illuminated the central LED. The null meter was placed on top of the video monitor used to display lines.

White lines were displayed horizontally on a black background, using a monochrome video monitor. The monitor was placed approximately $50 \mathrm{~cm}$ from the bridge of the subject's nose, at a comfortable viewing level (about chest high). Preliminary experiments showed that monitor distance did not have a significant effect on finger span matching for distances from 50 to $150 \mathrm{~cm}$. The lines were two pixels thick (out of 480 pixels available) and could be from 2 to 640 pixels long $(0.067-21.5 \mathrm{~cm})$, although subjects did not use lines longer than about $14 \mathrm{~cm}$. The length of the line was either set by the experimenter or controlled by the subject via a large, unmarked knob connected to a digital rotary encoder. The encoder had a resolution of $0.052^{\circ}$, and one full turn of the encoder produced a line the full width of the screen.

Ten "nominal" line lengths were used in the $S_{\text {free }} \rightarrow L$ experiment, ranging from $15 \%$ to $85 \%$ of the maximum span for each subject in steps of $7.8 \%$. Each subject, then, viewed a different set of lines. For example, the subject with the largest maximum span $(15.6 \mathrm{~cm})$ viewed lines that ranged in length from 2.3 to $13.3 \mathrm{~cm}$, but the subject with the smallest maximum span $(6.6 \mathrm{~cm})$ only viewed lines from 1.0 to $5.6 \mathrm{~cm}$. The length of the line presented in any particular trial, moreover, was selected at random within a window that was $\pm 5 \%$ of the maximum span about each nominal length. The random variations prevented subjects from memorizing and responding habitually to the different nominal lengths. A line was presented on the monitor in each trial, and, starting from zero span, the subjects opened their fingers to produce a span equal to the length of the line. It was helpful for many subjects to imagine picking up the line segment between the thumb and the index finger. The subject pressed a button with the opposite hand when satisfied with the match, and then held the span for a second until signaled to relax by a beep. The span was sampled for $1 \mathrm{sec}$ following the buttonpress, and the average and the standard deviation were calculated and stored. Each session consisted of 6 sets of trials, where every set consisted of 5 blocks containing one presentation of all 10 nominal line lengths, for a total of 300 trials. There was a delay of $2 \mathrm{sec}$ between the end of one trial and the start of the next, the blocks were separated by a $30-\mathrm{sec}$ rest period, and the sets were separated by $3 \mathrm{~min}$. The entire session lasted approximately $1 \mathrm{~h}$.

The complementary experiment, in which subjects adjusted the length of the line segment to match a preset finger span, was performed similarly. The subject produced and held one of 15 nominal finger spans as indicated by the null meter in each trial. The nominal spans again ranged from $15 \%$ to $85 \%$ of the maximum span, with a random variation of $\pm 5 \%$ (of the maximum span) about the nominal lengths. Once the target span was achieved (as indicated by the green LED in the meter), the subject used the rotary encoder to adjust the length of the line segment, starting at a length of $0.07 \mathrm{~cm}$ ( 2 pixels) to make a match. The subject then pressed a button with the hand used to turn the encoder, and the span was recorded as described above. The span measurement was not accepted if the green LED was not on at the time the button was pressed. The session consisted of 5 sets of trials. In each set, the 15 nominal spans were presented in 3 blocks of 5 trials each $(75$ trials total). Again, there was a $2-\sec$ pause between the end of one trial and the beginning of the next, a $30-\mathrm{sec}$ rest between blocks, and a 3-min rest between sets. Each session lasted about an hour.

The direct numerical estimation sessions were similar to the $L \rightarrow S_{\text {free }}$ sessions, except that once the finger span was achieved, the subject reported an estimate of the finger span (using inches or centimeters) instead of adjusting the length of a line.

\section{Experiment 2}

Twelve new subjects performed the second experiment, which was designed to test for differences in the perception of span depend- 
ing on whether the fingers contacted an object or not. Furthermore, the protocol was altered from the first experiment in order to make the procedure and results more directly comparable to data from previous experiments (in particular, R. Teghtsoonian \& M. Teghtsoonian, 1970). Subjects again adjusted line length to match finger span, but the thumb and the index finger were free in one set of trials and touching blocks in another set. In both sets, the same nine spans were used for all subjects $(0.50,1.00,1.50,2.00,3.50$, $4.50,6.00,7.50$, and $9.00 \mathrm{~cm}$ ), identical to the lengths used previously by R. Teghtsoonian and M. Teghtsoonian. The order in which the two sets were completed was counterbalanced across subjects.

The nine blocks that subjects touched were made from hardwood, with a height of $15.2 \mathrm{~cm}$ and a depth of $1.27 \mathrm{~cm}$ (as in R. Teghtsoonian \& M. Teghtsoonian, 1970), and with widths as listed above (within $0.01 \mathrm{~cm}$ ). The subject started each trial with his $/$ her fingers opened to their maximum span. The experimenter then touched one of the blocks to the subject's thumb and held the block loosely against the table. The subject closed the index finger, and the experimenter allowed the block to be reoriented if necessary, so that contact was made against the appropriate faces. The experimenter then held the block firmly against the table. The subjects were not permitted to lift or move the blocks. A similar procedure was used by Stevens and Stone (1959) and Chan et al. (1990). Once the subject's fingers were in contact with the block, the subject adjusted the line length to match the perceived block width. The nine blocks were presented in random order twice ( 18 trials). Neither the smallest block nor the largest block, however, was ever presented first in both sets of 9 trials. The subject did not see the blocks prior to the matching experiment.

Free finger spans were produced as described above, using the LEDs and a newer motion analysis system (OptoTrac, Northern Digital Inc., Waterloo, ON) that provided much more accurate measurements than did the SelSpot system used in Experiment 1. The nine spans were presented in random order twice. To match the motion required for touching the blocks, the subjects were instructed to start each trial at their maximum span and to close to the desired separation, indicated on the null meter as in Experiment 1 . The lines on the computer monitor started at the minimal length $(0.07 \mathrm{~cm})$ as before.

\section{RESULTS}

\section{Experiment 1}

Four matching functions are plotted for each subject in Figure 4 (the repeated session and the dead-reckoning results are not plotted). Circles represent $S_{\text {free }} \rightarrow L$ matches and triangles are $L \rightarrow S_{\text {free }}$ matches; the open points are data from the left hand, and closed points are from the right. Perhaps the most obvious result is the variability within and across subjects. Subjects 8 and 9 , for example, were reasonably consistent across all four conditions, but the shapes of the $L \rightarrow S_{\text {free }}$ and $S_{\text {free }} \rightarrow L$ functions were distinctly different for Subjects $1,2,3,10,11$, and 12. Of these, all but Subject 2 had accelerating $S_{\text {free }} \rightarrow L$ functions with spans usually underestimated by line length. Subjects 4 and 5 had three consistent sessions and one disparate session, but the latter was not under the same condition for both subjects. Other results were mixed.

Also shown in Figure 4 are power functions fit to the matching data, using the following equations:

$$
\begin{gathered}
S_{\text {free }}=a^{\prime} L^{b^{\prime}}+c \\
L=a^{\prime}\left(S_{\text {free }}-c\right)^{b^{\prime}}
\end{gathered}
$$

where

$$
\begin{aligned}
& a^{\prime}=10^{a} \\
& b^{\prime}=\tan (b) .
\end{aligned}
$$

The functions were fit to the matches averaged across repetitions of the nominal target line lengths or spans, and the standard errors $\sigma_{i}$ of each point were introduced as explicit weights by using the following objective function:

$$
\phi=\Sigma\left[y_{i}-f\left(x_{i}\right)\right]^{2 /} \sigma_{i}^{2} .
$$

The value of the independent variable $x_{i}$ in Equation 7 was also the average across repetitions of the $i$ th nominal length (with superimposed random variations as described above). All fits were performed using a Marquardt algorithm that adjusted $a, b$, and $c$ to minimize $\phi$.

The offset $c$ was introduced to account for potential errors made in calibrating the finger span readings. That is, the zero span was measured only once at the beginning of each session and was used throughout. Any random errors made in measuring the zero span were propagated throughout the experiment as a constant offset. The offset could not be introduced into the fit without any restrictions on its range of values, however. In some cases, it was possible for the three parameters to covary, yielding functions that failed to converge, with arbitrarily large and negative values of $c$, small $a$, and large $b$. The offset was introduced by including a data point at $x=0, y=0$, with a weight equal to the standard deviation of all re-zero measurements $(0.403 \mathrm{~cm})$. Since only one zero measurement was made, there was a greater uncertainty about the location of the intercept than about the location of the means at the other spans or lengths; so the standard deviation was used rather than the standard error. The weighted residual error from this term was $a l$ ways calculated in the direction of span - even for Equation 4 , where the dependent variable is line length.

The offset procedure can be confirmed, at least in part, by comparing the distribution of observed re-zero errors $(M=0.083 \mathrm{~cm}, S D=0.403 \mathrm{~cm})$ to the values obtained for $c$. Excluding one extreme value $(c=-1.99)$, the offsets had a mean of $-0.001 \mathrm{~cm}$ and a standard deviation of $0.488 \mathrm{~cm}$. The means were not statistically different (unpaired $t$ test, $p>.1)$, but the variances were $(F$ test, $p=.04)$. The values for both covered a range from -1.5 to $1.5 \mathrm{~cm}$. The procedure of introducing the offset, then, did produce values comparable to the re-zero errors, as expected.

The other two parameters estimated for each fit were $a$, the antilog of the scale factor, and $b$, the arctangent of the exponent. These parameters were estimated rather than the scale $a^{\prime}$ and exponent $b^{\prime}$ directly in order to simplify subsequent analysis of the parameter values. ${ }^{3}$ In all the discussions that follow, statistics are performed on the antilogs and arctangents ( $a$ and $b$ ), though summary values will be expressed traditionally as scales and exponents $\left(a^{\prime}\right.$ and $\left.b^{\prime}\right)$.

One question of immediate interest is whether the matching functions produced by individual subjects were stable or whether they varied systematically across the different sessions. As a first test, the parameters $a$ and $b$ were compared across the first and fifth (repeated) ses- 


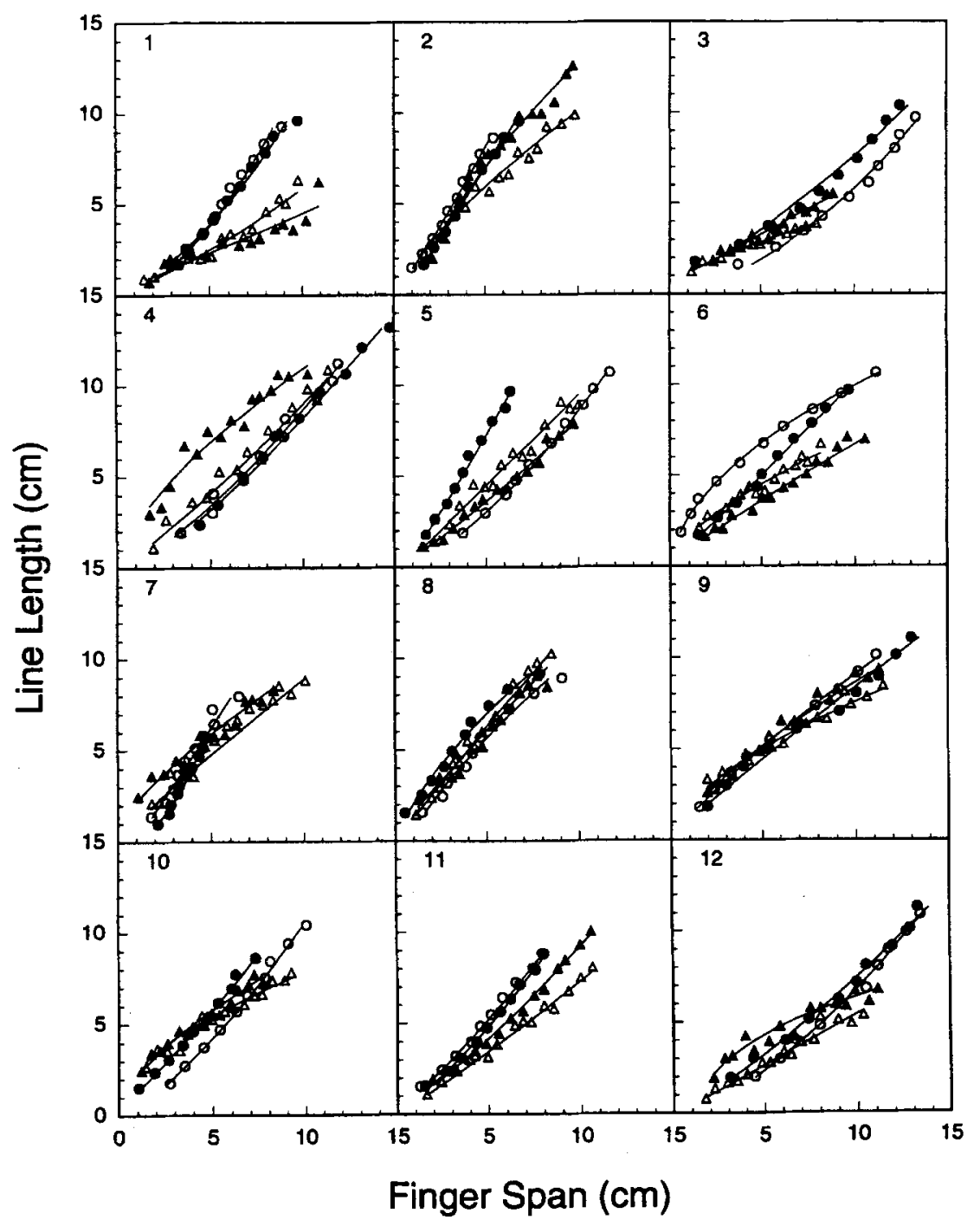

Figure 4.Cross-modal matching data from each subject in the first experiment of the present study. Finger spans were free; that is, they were made without contacting an object. Circles are $S_{\text {free }} \rightarrow L$ matches, and triangles are $L \rightarrow S_{\text {free }}$ matches. Open points are data from the left hand, and closed points are from the right. Each point is the average of 30 trials $\left(S_{\text {free }} \rightarrow L\right)$ or 5 trials $\left(L \rightarrow S_{\text {free }}\right)$. The lines are the best-fitting power functions as described in the text.

sion-that is, under identical experimental conditions. The correlations were insignificant $(a, r=.341, p=.287$; $b, r=.507, p=.094)$, and Bartlett's test of sphericity was not violated in either case ( $p=.477$ and .169 , respectively). The correlations were also insignificant if the data were separated according to task $\left(S_{\text {free }} \rightarrow L\right.$ or $\left.L \rightarrow S_{\text {free }}\right)$. The lack of correlation suggests that the parameter values were not repeatable for the same subject performing the same task in different sessions.

It is not too surprising, then, that the parameters also were not correlated across different experimental conditions within the same subject. Multiple correlations were calculated for $a$ and $b$, between all four combinations of the two matching directions and two hands (dominant and nondominant). The correlation matrices are given in Table 2. The two strongest correlations for each parameter were between the dominant and nondominant hands in the same matching direction. None of the correlations were statistically significant, however.

The lack of correlation, however, does not eliminate the possibility that there is a difference in the average value of the parameters in the different experimental conditions. To test this possibility, the parameters were analyzed first by using a repeated measures multivariate analysis of covariance (MANCOVA) with $a$ and $b$ as the dependent variables (which are highly correlated to each other, thus mandating the multivariate approach), matching direction and hand as within-subjects factors, and maximum span as a covariate. 
Table 2

Correlation Matrices for $a$ (Antilog of Scale Factor $a^{\prime}$ ) and $b$ (Arctangent of Exponent $b^{\prime}$ ) Across Match Direction and Hand

\begin{tabular}{|c|c|c|c|c|}
\hline & \multicolumn{2}{|c|}{ Nondominant } & \multicolumn{2}{|c|}{ Dominant } \\
\hline & $S_{\text {free }} \rightarrow L$ & $L \rightarrow S_{\text {free }}$ & $S_{\text {free }} \rightarrow L$ & $L \rightarrow S_{\text {free }}$ \\
\hline$a$ & 1.000 & $\begin{array}{r}-0.290 \\
1.000\end{array}$ & $\begin{array}{r}0.511 \\
-0.227 \\
1.000\end{array}$ & $\begin{array}{r}-0.317 \\
0.410 \\
-0.408 \\
1.000\end{array}$ \\
\hline$b$ & 1.000 & $\begin{array}{r}-0.176 \\
1.000\end{array}$ & $\begin{array}{r}0.501 \\
-0.009 \\
1.000\end{array}$ & $\begin{array}{r}-0.205 \\
0.222 \\
-0.101 \\
1.000\end{array}$ \\
\hline
\end{tabular}

None of the effects or their interactions was significant. The largest effect was that of hand on $a(p=.160)$. In order to include an effect of subject, and since the correlations within subjects were so poor and the interaction terms of the complete model were so weak, the main factors (subject, hand, and direction) were included in a non-repeated measures MANCOVA with maximum span as a covariate. In this case, maximum span had a significant multivariate effect $(p=.024)$, but only for its univariate effect on $b$. This effect was due primarily to the contributions of two subjects ( 6 and 11$)$ who also had the only significant individual correlations between $b$ and their maximum spans $\left(r^{2}=\right.$ .956 and .757 , respectively). If their data are removed from the analysis, the univariate effect on $b$ becomes insignificant $(p=.270)$. In fact, removing a single session for Subject 6 where $b$ had an extreme value (1.071) reduces the significance to $p=.088$. Therefore, it seems safe to conclude that maximum span had, at best, a weak and inconsistent effect on the parameters.

Given the absence of any significant within-subject correlation or a significant effect of maximum span, hand, or subject, it is appropriate to pool the parameter estimates for each direction, as listed in the top two rows of Table 3. (The third row listing the $L \rightarrow S_{\text {free }}$ "avg" parameters is discussed later.) The (weighted) average values of $a$ were $0.20 \pm 0.037$ (mean \pm standard error) and $0.11 \pm$ 0.013 for the $S_{\text {free }} \rightarrow \mathrm{L}$ and L $\rightarrow S_{\text {free }}$ experiments, respectively; and the average values of $b$ were $0.74 \pm 0.017$ and $0.72 \pm 0.010$. Table 3 also lists the parameters in their conventional forms (scale factor $a^{\prime}=10^{a}$ and exponent $b^{\prime}=$ $\tan b$; see Equations 3-6), along with their upper and lower $95 \%$ confidence limits. Ali the parameters were different from 1 , as required for equal matching (i.e., $L=S_{\text {free }}$ ).

A similar analysis can be applied to the dead-reckoning data. The results were fit using the same equation used for the $L \rightarrow S_{\text {free }}$ experiment, and the parameters are listed in the fourth row of Table 3 . The weighted average results were: $a=0.093 \pm 0.018$ and $b=0.76 \pm 0.012$, so that $a^{\prime}=1.2$ and $b^{\prime}=0.94$. Both parameters are significantly different from 1 .

\section{Experiment 2}

Two matching functions are plotted for Subjects 13-24 in Figure 5. The open circles are line length matches to free $\operatorname{span}\left(L \rightarrow S_{\text {free }}\right)$, and the closed circles are matches to finger span constrained by touching the blocks $(L \rightarrow S)$. The functions are not markedly different from those for the first experiment.

The re-zero errors with the use of the OptoTrac system were small (measured three times per subject, $M=$ $-0.07 \mathrm{~cm}, S D=0.20 \mathrm{~cm}$ ), and fits made to the $L \rightarrow S_{\text {free }}$ data with an offset were not significantly better than fits without an offset for any subject $(p>.3)$. Therefore, the data for both free span and touched blocks were fit by using Equation 4 with $c=0$. The fits were also made to all of the raw data rather than the averages at each reference length because each length was only presented twice. The weighted average of the fit parameters across the 12 subjects are listed in Table 4. Again, the parameters were pooled across subjects since they were not correlated across matching conditions. The average values of $a$ were $0.20 \pm 0.031$ and $-0.076 \pm 0.038$ for the $L \rightarrow S_{\text {free }}$ and $L \rightarrow S$ experiments, respectively; and the average values of $b$ were $0.68 \pm 0.024$ and $0.83 \pm 0.020$.

The average parameters for the $L \rightarrow S_{\text {free }}$ functions were slightly but significantly different (or nearly so) between Experiments 1 and 2 (Tables 3 and $4 ; a, t=2.91, p=$ $.006 ; b, t=-1.97, p=.057)$, and are plotted for comparison in Figure 6 . The small difference could be due to changes in the protocol, or differences in the sample of subjects. The differences between the $L \rightarrow S_{\text {free }}$ and $L \rightarrow S$ functions from the second experiment, also plotted in Figure 6, were much larger and highly significant (Table 4; $a, t=5.57, p<.0001 ; b, t=-4.97, p<.0001$ ), suggesting a robust difference between matches made with versus without touching blocks.

\section{DISCUSSION}

There are two results of interest: the rather large variability in the matching functions within subjects and across subjects and the differences between the matching functions obtained when subjects judged free span or span while touching an object.

The average results of the first experiment are summarized in the upper panel of Figure 7; the uppermost line is the $L \rightarrow S_{\text {free }}$ function, and the lowest line is the $S_{\text {free }} \rightarrow L$ function. The two functions have opposing curvature and appear to be nearly symmetric. The symmetry suggests that true matches might lie somewhere between the two curves but are distorted by a bias that affects the adjustment of the matching stimulus regardless of the modality. The distortion may be due to the regression effect (Stevens \& Greenbaum, 1966; Stevens \& Guirao, 1963; or "compression bias"--see Poulton 1979, 1989) that has been observed by many investigators. The distortion is particularly apparent when one is measuring psychophysical functions for a modality by using magnitude estimation and magnitude production. The traditional practice is to estimate the unbiased exponent by taking the geometric mean of the ME and MP exponents (see, e.g., Collins \& Gescheider, 1989), or taking 


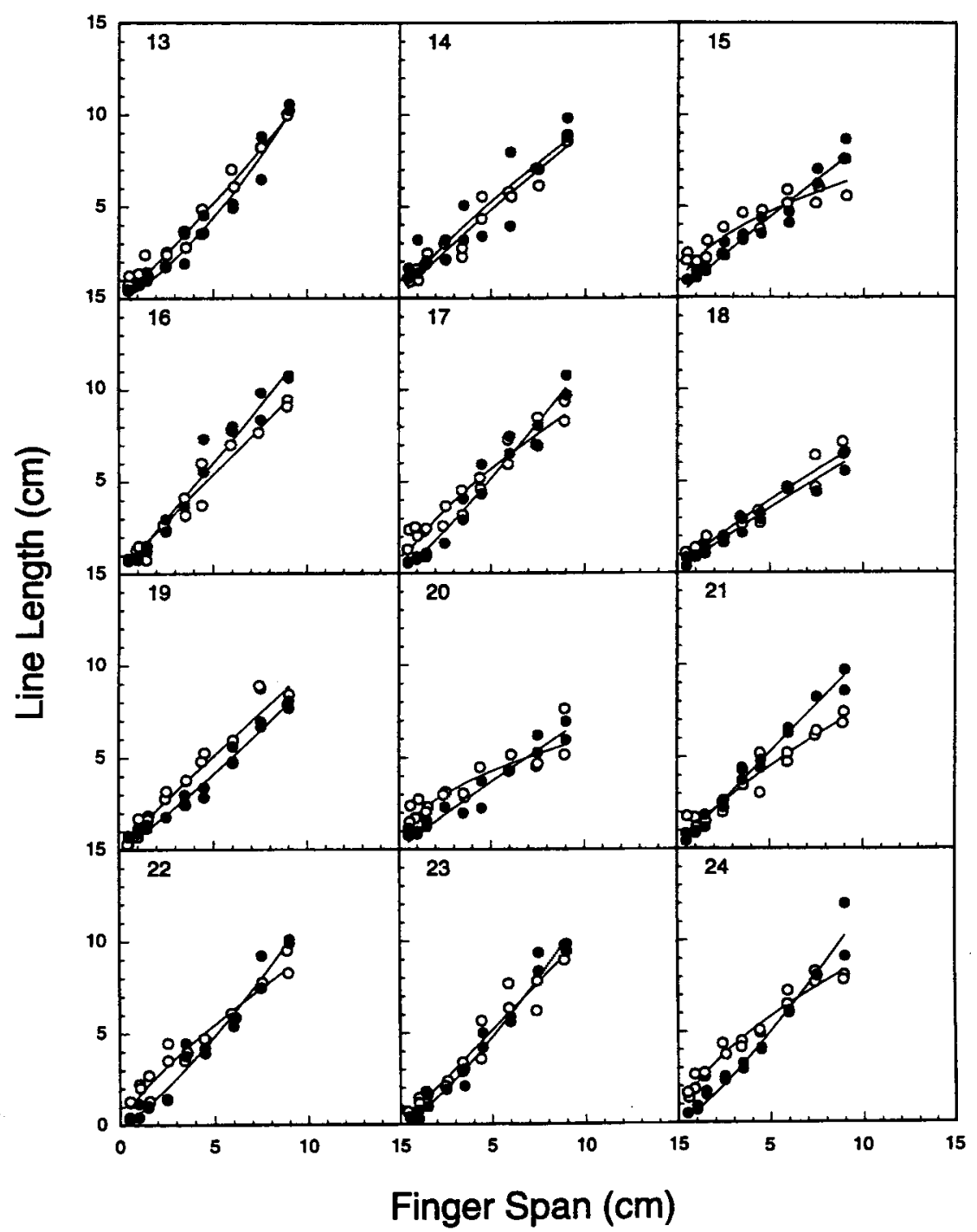

Figure 5. Cross-modal matching data from each subject in the second experiment of the present study. Line lengths were adjusted to match finger spans that were either free (open circles) or constrained by pinching blocks (closed circles). Each point is the result of a single trial. The lines are the best-fitting power functions as described in the text.

the geometrical means of the two functions ("magnitude balance"; Hellman \& Zwislocki, 1963). Since the actual stimulus values varied across subjects, I will use a functional, rather than an empirical, model of the bias effects, in which a function perturbs the sensation $\psi$ evoked by the reference stimulus. Suppose that perceived finger span and perceived line length can be described by the psychophysical functions:

$$
\psi=s S^{\sigma}
$$

and

$$
\psi=l L^{\lambda}
$$

respectively. We can predict the form of the cross-modal matching functions by equating the sensations $\psi$ :

$$
L=\left(\frac{s}{l}\right)^{\frac{1}{\lambda}} S^{\frac{\sigma}{\lambda}}=k_{l} S^{\varepsilon_{l}} .
$$

The matching bias is introduced by assuming that the reference sensation is distorted by a "biasing function," such that

$$
\hat{\psi}=b \psi^{\beta} \text {. }
$$

Therefore, the parameters of the experimentally observed cross-modal matching functions will also be biased:

$$
L=\left(\frac{b s^{\beta}}{l}\right)^{\frac{1}{\lambda}} S^{\frac{\sigma \beta}{\lambda}}=\hat{k}_{l} S^{\hat{\varepsilon}_{l}}
$$


Table 3

Average Free Span Matching Function Parameters, Standard Errors, and Upper and Lower 95\% Confidence Intervals

\begin{tabular}{|c|c|c|c|c|c|c|c|c|c|c|}
\hline \multirow[b]{2}{*}{ Match } & \multirow[b]{2}{*}{$a$} & \multirow[b]{2}{*}{$S E(a)$} & \multirow[b]{2}{*}{$10^{a}$} & \multicolumn{2}{|c|}{ Interval } & \multirow[b]{2}{*}{$b$} & \multirow[b]{2}{*}{$S E(b)$} & \multirow[b]{2}{*}{$\tan b$} & \multicolumn{2}{|c|}{ Interval } \\
\hline & & & & Lower & Upper & & & & Lower & Upper \\
\hline$S_{\text {free }} \rightarrow L$ & 0.20 & 0.037 & 1.6 & 1.3 & 1.9 & 0.74 & 0.017 & 0.92 & 0.86 & 0.99 \\
\hline$L \rightarrow S_{\text {free }}$ & 0.11 & 0.013 & 1.3 & 1.2 & 1.4 & 0.72 & 0.010 & 0.88 & 0.84 & 0.91 \\
\hline $\begin{array}{l}L \rightarrow S_{\text {free }} \\
\text { (unbiased) }\end{array}$ & & & 0.91 & & & & & 0.98 & & \\
\hline$N \rightarrow S_{\text {free }}$ & 0.093 & 0.018 & 1.2 & 1.1 & 1.4 & 0.76 & 0.012 & 0.94 & 0.89 & 1.0 \\
\hline
\end{tabular}

Table 4

Average Matching Function Parameters for Free Span and Touching Blocks, With Standard Errors and Upper and Lower $95 \%$ Confidence Intervals

\begin{tabular}{|c|c|c|c|c|c|c|c|c|c|c|}
\hline \multirow[b]{2}{*}{ Match } & \multirow[b]{2}{*}{$a$} & \multirow[b]{2}{*}{$S E(a)$} & \multirow[b]{2}{*}{$10^{a}$} & \multicolumn{2}{|c|}{ Interval } & \multirow[b]{2}{*}{$b$} & \multirow[b]{2}{*}{$S E(b)$} & \multirow[b]{2}{*}{$\tan b$} & \multicolumn{2}{|c|}{ Interval } \\
\hline & & & & Lower & Upper & & & & Lower & Upper \\
\hline$L \rightarrow S_{\text {free }}$ & 0.20 & 0.031 & 1.6 & 1.4 & 1.9 & 0.68 & 0.024 & 0.80 & 0.73 & 0.89 \\
\hline$L \rightarrow S$ & -0.076 & 0.038 & 0.84 & 0.71 & 1.0 & 0.83 & 0.020 & 1.1 & 1.0 & 1.2 \\
\hline
\end{tabular}

That is, the observed parameters ( $k$ and $\hat{\varepsilon}$ ) will not equal the unbiased parameters $(k$ and $\varepsilon)$. We can estimate the unbiased values, however, given both sets of biased crossmodal matching parameters $\left(L \rightarrow S_{\text {free }}\right.$ and $\left.S_{\text {free }} \rightarrow L\right)$. First, the unbiased exponent is given by the geometric mean of one biased exponent and the inverse of the second biased exponent:

$$
\varepsilon_{l}=\frac{\sigma}{\lambda}=\sqrt{\frac{\hat{\varepsilon}_{l}}{\hat{\varepsilon}_{s}}} .
$$

Also, the exponent of the bias function is given by

$$
\beta=\sqrt{\hat{\varepsilon}_{l} \hat{\varepsilon}_{s}^{-}} .
$$

Finally, the scale factor is equal to

$$
k_{l}=\left(\frac{s}{l}\right)^{\frac{1}{\lambda}}=\left(\frac{\hat{k_{l}}}{\hat{k}_{s}^{\frac{\sigma}{\lambda}}}\right)^{\frac{1}{\beta+1}}
$$

where we can substitute the values for $\sigma / \lambda$ and $\beta$ from the preceding equations, and use the observed scales of the two cross-modal matching functions.

The unbiased $L \rightarrow S_{\text {free }}$ parameters (Table 3, third row) were calculated with Equations 10-15 and the biased parameters (Table 3, first two rows), yielding $k_{l}=0.91$ and $\varepsilon_{l}=0.98$, so that the final estimate of the $L \rightarrow S_{\text {free }}$ matching function from the first experiment is

$$
L=0.91 S^{0.98} \text {. }
$$

The bias exponent is $\beta=0.90$, which is consistent with the common observation that the regression effect reduces the slope of the matching (or estimation) function.

Equation 16 implies that, if the model is correct, perceived line length and finger span are nearly proportional. The bias model cannot remove the nonlinearities when applied to the results from Jastrow (1886), yield- ing instead a strongly accelerating function with $k_{l}=$ 0.36 and $\varepsilon_{l}=1.4$. The bias-function exponent, $\beta=0.94$, agrees well with the result from the present experiment. Also, R. Teghtsoonian and M. Teghtsoonian (1970) used magnitude estimation (without a modulus) to measure psychophysical functions for both line length and finger span in the same subjects. By equating equal numbers, we can synthesize a cross-modal matching function (see, e.g., Mashhour and Hosman, 1968), yielding

$$
L=0.76 S^{1.1} \text {. }
$$

This constructed cross-modal matching function should be free of the compression bias as modeled above, pro-

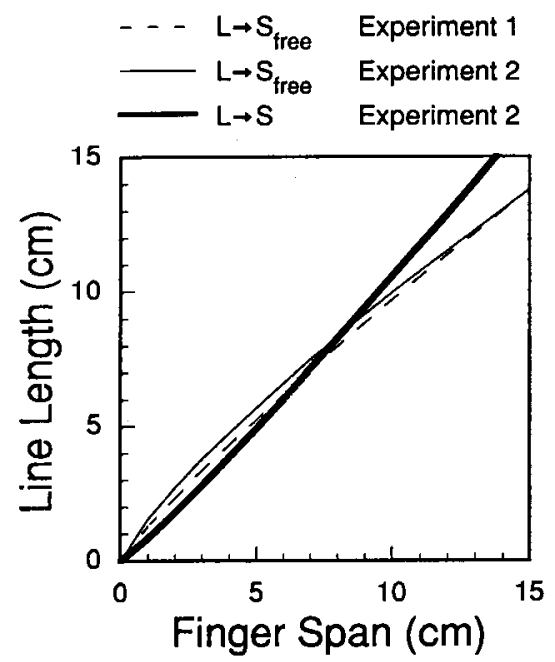

Figure 6. Average $L \rightarrow S_{\text {free }}$ functions from the first (dashes) and second (thin line) experiments. The function parameters are listed in Tables 3 and 4 . The two functions are slightly, but significantly, different. In contrast, the thick line is the $L \rightarrow S$ (pinching blocks) function from the second experiment, and has the opposite curvature of either $L \rightarrow S_{\text {free }}$ function. 
vided that the bias has affected magnitude estimates of span and length equally.

The results from the three comparable experiments do not agree; they are plotted in the lower panel of Figure 7. The present results suggest that span and length are perceived proportionally, whereas Jastrow (1886) and R. Teghtsoonian and M. Teghtsoonian (1970) found that length is an accelerating function of span with an exponent of 1.4 or 1.1 , respectively.

It is unlikely that this difference is due to the use of CMM as opposed to ME. Jastrow used a CMM protocol and measured the largest exponent, whereas the matches made in the present experiment yielded the smallest exponent. The ME data from R. Teghtsoonian and M. Teght-

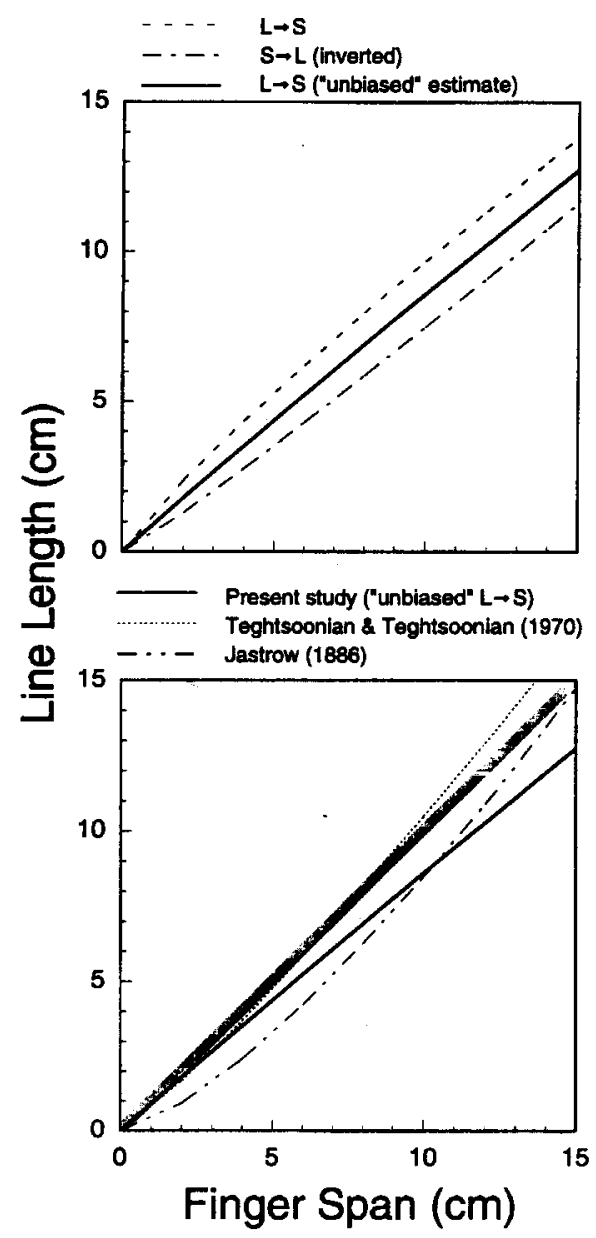

Figure 7. (Top) Average $L \rightarrow S_{\text {free }}$ (regular dashes) and $S_{\text {free }} \rightarrow L$ (irregular dashes) functions from the first experiment. The function parameters are listed in Table 3. The thick line is the unbiased $L \rightarrow S_{\text {free }}$ function as derived in the text. (Bottom) Comparison of the unbiased $L \rightarrow S_{\text {free }}$ from 6a (thick line) with the cross-modal matching function synthesized from the magnitude estimation functions of $R$. Teghtsoonian and $M$. Teghtsoonian (1970; small dashes), and the unbiased $L \rightarrow S$ function derived from the $S \rightarrow L$ and $L \rightarrow S$ functions of Jastrow (1886, irregular dashes). Gray line represents equality of span and line length. soonian (1970) produced a mildly accelerating function, but the dead-reckoning function was slightly, but significantly, decelerating. The instructions in the latter were to actually estimate the length in physical units, not to use a "reasonable" number or a number related to a modulus. However, since previous magnitude estimation results have produced numerical ratings close to physical dimensions in inches (M. Teghtsoonian \& R. Teghtsoonian, 1965; R. Teghtsoonian \& M. Teghtsoonian, 1970), the actual difference in estimation procedures is relatively small.

The differences, alternatively, may be due to the way finger span was produced and measured. R. Teghtsoonian and M. Teghtsoonian (1970) used wooden blocks held aloft between the thumb and the index finger, which also provided weight cues (Jones, 1983). Jastrow (1886) also used wooden blocks that were pinched (but not lifted) when line length was matched to the span (see also Chan et al., 1990; Stevens \& Stone, 1959). Lines were either selected from a fixed set, or indicated by a mark made on a ruler. In the complementary experiment, span was produced and measured with a pair of parallel plates attached to sliding carriages. The carriages were set in diverging tracks, so movement of the carriages along the tracks was converted to changes in the separation of the plates. The plates were pinched, and the subject had to move the carriages along the track to adjust the span between the plates. No objects were handled in the first experiment of the present study, and no arm or hand movements were required either to produce the span or to adjust the line length (apart from pronation and supination about the wrist).

The results of the second experiment support the proposition that perceived finger span depends on whether or not an object is touched. In this case, all details of the protocol were identical in two sets of trials in a single session for the same subject, except that blocks were pinched in one set and free span was used in the other set (with the order counterbalanced). Eleven of the 12 subjects had a larger exponent when touching the blocks, and the average exponent was significantly larger in the $L \rightarrow S$ condition (exponent $=1.1$ ) than for $L \rightarrow S_{\text {free }}(0.80)$. If the 12 subjects in the second experiment had the same average bias exponent as the first 12 subjects had $(\beta=0.90)$, then rough estimates of the unbiased $L \rightarrow S$ and $L \rightarrow S_{\text {free }}$ exponents are $1.1 / 0.90=1.2$ and $0.80 / 0.90=0.89$, respectively. These values are in reasonable agreement with the unbiased $L \rightarrow S$ exponents of R. Teghtsoonian and M. Teghtsoonian (1970) and Jastrow (1886), 1.1 and 1.4, respectively, and with the $L \rightarrow S_{\text {free }}$ exponent from the first experiment $(0.98)$.

The difference between the $L \rightarrow S_{\text {free }}$ functions of the first and second experiments should not be dismissed, but are probably attributable to differences in the two groups of subjects. The raw, biased exponents were 0.88 and 0.80 , respectively, and the difference just missed statistical significance. It was possible to compensate for subject biases in the first experiment by using the (presumably) 
symmetrically biased $S_{\text {free }} \rightarrow L$ results- but not so for Experiment 2. Using the estimated bias exponent from Experiment $1(\beta=0.90)$ to correct the results of Experiment 2 is, at best, an approximation. It may be prudent, though, to conclude that the exponent of the unbiased $L \rightarrow S_{\text {free }}$ function is 0.96 (Experiment 1) or slightly less.

A more robust and important difference exists between the psychometric functions for finger span with and without object contact. The difference can be summarized by synthesizing a CMM function for $S_{\text {free }}$ versus $S$ from the two line-length matching functions of Experiment 2 , yielding

$$
S_{\text {free }}=0.46 S^{1.4} \text {, }
$$

which accounts for most of the previous and current finger span matching and scaling results. There remains one discrepant study, however. Chan et al. (1990) measured $S \rightarrow S_{\text {free }}$ functions directly by using the index finger and thumb to touch blocks and by using the thumb and index finger or the thumb and middle finger of the opposite hand to make free span matches. When the index finger was used to generate the matching free span, the matching function was decelerating with an exponent of 0.79 (Equation 3)-which contradicts the conclusion made above (Equation 18). ${ }^{4}$ The matching function with the middle finger, though, had an exponent of 1.1 , which agrees better with the present results. Chan et al. attribute the downward curvature for matching with the index finger to a response compression induced by the difficulty in achieving large spans (up to $13 \mathrm{~cm}$ ) between the thumb and the index finger. The difficulty is reduced and the exponent is increased by using the longer middle finger. Higher exponents would be expected in the present experiments, therefore, since the subjects never produced spans that they felt were uncomfortably large. It is also possible that regression bias reduces the exponents reported by Chan et al., and that the unbiased values would be higher.

The second result of interest is the variability of the matching functions within and across subjects, even though the matching task was, perhaps, as simple as one can perform between two sensory continua. Several investigators have studied the individual differences among subjects performing magnitude estimation. Of particular interest are the results of Collins and Gescheider (1989), who found that the ME exponents for loudness and line length were correlated within a group of subjects (but see M. Teghtsoonian and R. Teghtsoonian, 1971, 1983), so that the standard deviation of each exponent (adults: loudness - 0.155 , line length -0.582 ), was larger than the standard deviation of their ratio (loudness/line length$0.039)$. The variability of the ratio was comparable to that of direct cross-modal matching exponents $(S D=0.054)$. Gescheider $(1988,1993)$ suggests that an individual may assign numbers in a unique way that is stable across sessions and even sensory continua. The results from the first experiment do not support extending that hypothesis to span and length matching. There was no significant correlation between parameters for the identical conditions repeated in two sessions, nor were the parameters correlated across the different matching conditions. Even though the subjects were making cross-modal matches, the standard deviations of the exponents ( $L \rightarrow S_{\text {free }} ; 0.242$, $\left.S_{\text {free }} \rightarrow L: 0.299\right)$ were comparable to those of the ME exponents of Collins and Gescheider (1989).

The results in general suggest that perceived line length and free finger span are proportional, but not equal. Free finger span is perceived as being about $90 \%$ of the actual length. Finger span is an accelerating function of actual span, however, if the fingers contact an object, as has been found by previous investigators (most notably, R. Teghtsoonian and M. Teghtsoonian, 1970; and Jastrow, 1886). The basis for this difference is not known, but it may be similar to "kinesthetic illusions" in which differences in applied force affect perceived position, and conversely (Mai, Schreiber, \& Hermsdörfer, 1991; Roland \& Ladegaard Pedersen, 1977; Rymer \& D'Almeida, 1980; Watson, Colebatch, \& McCloskey, 1984). Alternatively, the two tasks may reflect qualitative rather than quantitative differences in the perceptual continua. Stevens and Stone (1959) correctly suggested that finger "span" could be sensed via finger position, presumably a metathetic continuum, rather than by the actual finger-thumb separation. Their category scaling and discrimination results, however, show that finger span for touched blocks is prothetic. Pinching blocks, however, allows span to be defined or perceived as an unequivocal extensive property of an object. There is a greater possibility that subjects could make judgments of free span based on finger position. It would be curious, though, for subjects in the same session to judge the distance between the finger and thumb when an object was present, but the position of the finger and thumb in the absence of an object. Finally, there is some ambiguity in identifying the external physical correlate of free span. That is, it is not certain that a subject's perception of span (if prothetic) is referenced to the points on the fingertips at which span was measured. In sum, there are several possible explanations for the differences between perceptions of span with and without object contact. The effect of contact force can be studied easily by repeating the experiment with different load conditions. Likewise, it would be straightforward to use category scaling and discrimination to help resolve whether free span is prothetic or metathetic. Identifying the appropriate physical reference for free span, however, promises to be a more challenging problem.

\section{REFERENCES}

Anderson, N. H. (1974). Algebraic models in perception. In E. C. Carterette \& M. P. Friedman (Eds.), Handbook of perception (Vol. 2, pp. 215-298). Dordrecht: D. Reidel.

BERGLUND, B., \& OLSSON, M. J. (1993). Odor-intensity interaction in binary and ternary mixtures. Perception \& Psychophysics, 53, 475482 . 
Bevington, P. R. (1969). Data reduction and error analysis for the physical sciences. New York: McGraw-Hill.

Chan, T.-C., Carello, C., \& Turvey, M. T. (1990). Perceiving object width by grasping. Ecological Psychology, 2, 1-35.

Collins, A. A., \& Gescheider, G. A. (1989). The measurement of loudness in individual children and adults by absolute magnitude estimation and cross-modality matching. Journal of the Acoustical Society of America, 85, 2012-2021.

Davidon, R. S., \& MATHER, J. H. (1966). Cross-modal judgments of length. American Journal of Psychology, 79, 409-418.

Ekman, G., Berglund, B., Berglund, U., \& Lindvall, T. (1967). Perceived intensity of odor as a function of time of adaptation. Scandinavian Journal of Psychology, 8, 177-186.

Gescheider, G. A. (1988). Psychophysical scaling. Annual Review of Psychology, 39, 169-200.

GESCHEIDER, G. A. (1993). What is absolute about absolute scaling? In R. T. Verrillo (Ed.), Sensory research: Multimodal perspectives (pp. 211-232). Hillsdale, NJ: Erlbaum.

Gogel, W. C., Wist, E. R., \& HARKer, G. S. (1963). A test of the invariance of the ratio of perceived size to perceived distance. American Journal of Psychology, 76, 537-553.

Hellman, R. P., \& Zwislocki, J. J. (1963). Monaural loudness function of a 1000 -cps tone and internal summation. Journal of the Acoustical Society of America, 35, 856-865.

JASTROW, J. (1886). The perception of space by disparate senses. Mind, $11,539-554$.

JONEs, B. (1983). Psychological analyses of haptic and haptic-visual judgements of extent. Quarterly Journal of Experimental Psychology, 35A, 597-606.

Mai, N., Schreiber, P., \& Hermsdörfer, J. (1991). Changes in perceived finger force produced by muscular contractions under isometric and anisometric conditions. Experimental Brain Research, 84, 453-460.

Mashhour, M., \& Hosman, J. (1968). On the new "psychophysical law": A validation study. Perception \& Psychophysics, 3, 367-375.

Poulton, E. C. (1979). Models for biases in judging sensory magnitude. Psychological Bulletin, 86, 777-803.

Poulton, E. C. (1989). Bias in quantifying judgments. Hillsdale, NJ: Erlbaum.

Roland, P. E., \& LadegaArd-Pedersen, H. L. (1977). A quantitative analysis of sensations of tension and kinæsthesia in man: Evidence for a peripherally originating muscular sense and for a sense of effort. Brain, 100, 671-692.

Rymer, W. Z., \& D'Almeida, A. (1980). Joint position sense: The effects of muscle contraction. Brain, 103, 1-22.

Stanley, G. (1966). Haptic and kinesthetic estimates of length. Psychonomic Science, 5, 377-378.

STEVENS, S. S. (1969). On predicting exponents for cross-modality matches. Perception \& Psychophysics, 6, 251-256.

Stevens, S. S., \& Galanter, E. H. (1957). Ratio scales and category scales for a dozen perceptual continua. Journal of Experimental Psychology, 54, 377-411.

Stevens, S. S., \& Greenbaum, H. B. (1966). Regression effect in psychophysical judgment. Perception \& Psychophysics, 1, 439-446.

STEVENS, S. S., \& GUIRAO, M. (1963). Subjective scaling of length and area and the matching of length to loudness and brightness. Journal of Experimental Psychology, 66, 177-186.

STEVens, S. S., \& STone, G. (1959). Finger span: Ratio scale, category scale, and JND scale. Journal of Experimental Psychology, 57, 91-95.

Teghtsoonian, M., \& Teghtsoonian, R. (1965). Seen and felt length. Psychonomic Science, 3, 465-466.

Teghtsoonian, M., \& Teghtsoonian, R. (1971). How repeatable are Stevens's power law exponents for individual subjects? Perception \& Psychophysics, 10, 147-149.

Teghtsoonian, M., \& Teghtsoonian, R. (1983). Consistency of individual exponents in cross-modal matching. Perception \& Psychophysics, 33, 203-214.

Teghtsoonian, R., \& Teghtsoonian, M. (1970). Two varieties of perceived length. Perception \& Psychophysics, 8, 389-392.

VERRILLO, R. T. (1983). Stability of line-length estimates using the method of absolute magnitude estimation. Perception \& Psychophysics, 33, 261-265.

Watson, J. D. G., Colebatch, J. G., \& MCCloskey, D. I. (1984). Effects of externally imposed elastic loads on the ability to estimate position and force. Behavioral Brain Research, 13, 267-271.

\section{NOTES}

1. The data provided by Berglund and Olsson were the coordinates of smooth curves drawn by eye through the average data for each of 8 subjects. Each curve represented the average of 10 sets of trials, and the coordinates were given for every $1 \%$ step in the target percentage. Finger spans were originally generated in $10 \%$ increments from $5 \%$ to $95 \%$ of the maximum, but $I$ used the coordinates at $10 \%$ intervals from $10 \%$ to $90 \%$ to make the data commensurate with those of Ekman et al. (1967).

2. The optical tracking system calculated the LED separation from independent measurements of the LED locations in space. Consequently, it was possible for errors in the measured position to yield negative values for the calculated span.

3. Once the offset is removed from Equations 3 and 4, we can take the logarithms of both sides, giving:

$$
\log S=\log a^{\prime}+b^{\prime} \log L=a+\tan (b) \log (L)
$$

and

$$
\log L=\log a^{\prime}+b^{\prime} \log S=a+\tan (b) \log (S) .
$$

Since $a$ is an intercept-an additive factor-it is appropriate to perform statistics on it directly (e.g., take the arithmetic mean, etc.). More importantly, the standard errors generated by the fitting algorithm are truly symmetric about the mean estimate and may be used to find weighted averages directly (i.e., without a transformation) from the formula (Bevington, 1969):

$$
\mu(a)=\Sigma\left(a / \sigma_{a}^{2}\right) / \sum\left(1 / \sigma_{a}^{2}\right) .
$$

Similarly, using the arctangents of the exponents (i.e., the angles corresponding to the slopes of the power functions in logarithmic coordinates) allows conventional averaging. (Consider, for instance, that the average of $0^{\circ}$ and $90^{\circ}$ is $45^{\circ}$, whereas the arithmetic average slope is infinite, and the geometric average slope is undefined.)

4. Chan et al. (1990) fit their data with quadratic functions rather than power functions and allowed for a nonzero intercept. The intercept appears as a nonzero free span at zero block width. Since they measured free span with a ruler, it is not clear how a nonzero intercept could arise unless the technique produced a systematic over- or underestimation of span at all block widths. The intercept is preserved here to be consistent with the original analysis, but the fits should be interpreted with caution.

\section{APPENDIX Algebraic Synthesis of Matching Functions}

As in many previous treatments, I will manipulate and synthesize cross-modal matching functions algebraically. First, I assume that the continua of line length $(L)$, finger span $(S)$, and number $(N)$ have psychometric functions given by

$$
\begin{aligned}
& \psi=l L^{\lambda}, \\
& \psi=s S^{\sigma}, \\
& \psi=n N^{v},
\end{aligned}
$$

where $\psi$ is the sensation magnitude. Cross-modal matching of line lengths to finger spans that evoke identical sensation magnitudes should produce a CMM function given by

$$
L=\left(\frac{s}{l}\right)^{\frac{1}{\lambda}} S^{\frac{\sigma}{\lambda}}
$$


The $S \rightarrow L$ function is simply the inverse-that is,

$$
S=\left(\frac{l}{s}\right)^{\frac{1}{\sigma}} L^{\frac{\lambda}{\sigma}} .
$$

As a result, the $S \rightarrow L$ function should have an exponent that is the reciprocal of the $L \rightarrow S$ exponent. Magnitude estimation can also be treated as a matching of numbers to another continuum, so that

$$
N=\left(\frac{s}{n}\right)^{\frac{1}{v}} S^{\frac{\sigma}{v}},
$$

$$
N=\left(\frac{l}{n}\right)^{\frac{1}{v}} L^{\frac{\lambda}{v}} .
$$

Note that the exponents of the $N \rightarrow S$ (magnitude estimation of span ) and $L \rightarrow S$ functions ( $\sigma / v$ and $\sigma / \lambda$, respectively) will be equal only if $v=\lambda$. In fact, it has been found in many studies that magnitude estimates of line length are proportional to line length (e.g., Stevens \& Galanter, 1957; Stevens \& Guirao, 1963; Mashhour \& Hosman, 1968; M. Teghtsoonian \& R. Teghtsoonian, 1965; R. Teghtsoonian \& M. Teghtsoonian, 1970; Verrillo, 1983), which requires that $\lambda / v=1$ (Equation A3b), so that $v=\lambda$.

(Manuscript received December 28, 1993;

revision accepted for publication November 22, 1994.) 